\title{
Analytical Background for Developing a Body of Knowledge for Cartography
}

\author{
Harold Moellering \\ Dept. of Geography, Ohio State University, Columbus, OH , 43210, U.S.A., Email: moellering.1@osu.edu
}

\begin{abstract}
At the 2011 ICC meetings in Paris, Moellering (2011) discussed the ICA Research Agenda, looked to the future, and recommended that the ICA move to develop a formal Body of Knowledge for the field of Cartography. In the last few years, the ICA leadership has recognized this need, and has organized an effort to develop a systematic Body of Knowledge (BoK) for the field of Cartography.

In order to develop a systematic and comprehensive BoK for Cartography, it is important to include all aspects of the field: conceptual, conventional, perceptual, virtual, production, quantitative and analytical. In this milieu many of the long-standing components of cartography exist in the Surface Structure of the field, meaning that they are directly and visually observed. Most of those components have been recognized for many decades, even centuries.
\end{abstract}

However, the analytical and mathematical components of cartography exist in the Deep Structure of the field. These are associated with the data, information, and metadata that underlie the standard visualizations process. These quantitative and analytical components in cartography mostly are relatively new, being developed in recent decades.

Essentially these new analytical components in the Deep Structure can be considered the new half of the field of cartography, and it is essential that they be included in a full definition of the BoK for cartography. This paper will discuss these conceptual analytical components of Cartography and relate them to the evolving Body of Knowledge for the field.

Keywords: Analytical Cartography, Cartography, Body of Knowledge, BoK, Geospatial Theory, GIScience

\section{Introduction}

For almost as long as modern cartography has existed, many thoughtful members of the field have reflected and discussed the scope and content of the field. So each active researcher in the field has in mind an implicit understanding of what constitutes the knowledge base for the field. These views differ between researchers, and although there are apparent clusters of commonality, there is no overarching understanding of what constitutes the knowledge base for the field of cartography.

One way in which this effort has been focused is through the development of the ICA Research Agenda (Virrantaus, et.al. 2009). This effort was mostly inwardlooking and was focused on the work of the various ICA Commissions. However, Moellering (2011) reviewed this work in his paper for the Paris ICC 2011, and concluded that the ICA should rather be more outwardlooking, and with the view that the ICA should develop a formal definition of a Body of Knowledge for Cartography saying: "The ICA should move aggressively to bring together the implicit, although dispersed, concepts and theory in the field into an organized and systematic Body of Knowledge". These views were later published by Moellering (2012). In a similar light,
Buchroithner and Fernandez (2011) reviewed the epistemological situation of cartography, and stated:

"So far, cartography has experienced only little theoretical development. In such cases, methodologies and techniques are frequently mixed with a discipline's own scientific development, and techniques are thus considered as if they were genuine disciplinary knowledge. ..... In order to better understand the theoretical and technological dimensions of cartography, it is necessary to further identify aspects of its identity as an independent science. ..... If cartography wants to reach a wide-accepted more prominent scientific status, it needs, in addition, to its well-developed and up-to-date methodology, to create its own updated epistemological basis."

This kind of thinking leads directly towards the development of a Body of Knowledge for Cartography.

\section{Understanding the Nature of a Body of Knowledge (BoK)}

A straightforward definition of a Body of Knowledge (BoK) can be found in Wikipedia (2018): "A body of knowledge (BoK) is the complete set of concepts, terms and activities, and make up a professional domain, as 
defined by the relevant learned society or professional association. It is a type of knowledge representation by any knowledge organization.... A BoK is the accepted ontology for a specific domain.”

In this statement it is understood that any group initiating such an effort would clarify the epistemological basis for their work at the beginning. In essence, such an effort is taking the implicit understanding of the field of cartography by many individuals, and systematically pooling and organizing that into an explicit understanding of what constitutes the field of cartography. When that explicit understanding is formalized into a document, then it becomes a statement of a Body of Knowledge about a field or discipline.

\section{Sources for Items in a Cartographic Body of Knowledge (BoK)}

Such sources of items for a cartography BoK can be found in many places in the field. One can see the field of cartography as comprising three paradigms, which are conceptual modes of operation in a field. Here in cartography one can see three paradigms of cartographic operation:

- Mapping and Visualization Paradigm;

- Communication Paradigm;

- Analytical Paradigm.

From these three paradigms of cartography one would identify the various pieces of concepts, theory and professional practices that would become part of the BoK for the field. Clearly this will be a group activity of knowledgeable experts from various part of the field.

\section{Deep and Surface Structure Sources of Items for the Cartographic Body of Knowledge}

For over two and a half millennia cartographers have been making maps and visualizations as graphic representations of geographical space. Regardless of whether this is as a hard copy map or some kind of soft copy visualization (virtual map), items for the BoK emerge. This was defined by Nyerges (1991) as Surface Structure, as is shown by the following figure.

\section{Cartographic Surface Structure}

maps or visualizations directly viewed

\section{Cartographic Deep Structure}

map database, spatial information, and metadata

On the upper side of this diagram is Surface Structure which constitutes the standard mapping and visualization tasks and processes that have been part of the field of cartography for a long time. The hard copy map design, drawing, and construction are the oldest part of cartography going back more than twenty-five hundred years. The visualization part is much more recent in the last hundred years or so. So map display and visualization are the core of Surface Structure in cartography.

On the lower part of the diagram above one can see the Deep Structure of the data (Nyerges, 1991) from which the map/display image comes. Here, Deep Structure can represent a wide range of possibilities. For example, it could be a cartographic or spatial database. Or it could contain the scientific organization of how a map reader might perceive this image in the surface structure. So, perceptual structural organization is definitely part of the Deep Structure of any map or cartographic image. Another example of the Deep Structure shown here could be an ontological representation of the data/information shown on the map/display surface. Since most of this work has developed in the last several decades, one could characterize Deep Structure as the "New Half of Cartography".

\section{The Development of Analytical Cartography}

The modern origins of Analytical Cartography largely began with the work of Waldo Tobler in the $1960 \mathrm{~s}$ (Tobler, 1961), although one can trace ancient roots of the field to the 5th century $\mathrm{BC}$ when map projections were invented by the ancient Greeks. Tobler's initial primary goal was to "solve cartographic problems" in a quantitative/analytical way. With time his view of this goal matured to a point where the essence of his work was "to capture this theory"(Tobler, 1976).

\subsection{Early Developments in Analytical Cartography Via the Work of Tobler}

The work by Waldo Tobler was transformational in developing modern analytical cartography. The major threads of Tobler's work can be seen as:

\section{- 2-D Coordinate Map Transformations and Projections \\ - Cartograms \\ - Spatial Frequencies, Filters \& Neighborhoods \\ - Volumetric Pycnophlactic Transformations \\ - Resolution Elements - Resels \\ - Irregular Neighborhood Operators}

In the first two threads of 2-D map transformations, projections, and cartograms, Tobler's deep structure analytical and mathematical work resulted in surface structure map transformations, projections, and cartogram visualizations which fascinated the entire field of cartography beginning in the 1960s, and since have become standard tools for cartographic visualization.

The next four threads of spatial frequencies and neighborhood operators, pycnophlactic transformations, irregular neighborhood operators, and resolution elements 
(Resels) are analytical/mathematical deep structure operations in cartography that produce quantitative results. However, even with these and similar analytical operators, in many cases the results of the operations are realized in quantitative output and cartographic visualizations.

\subsection{More Recent Developments of Analytical Cartography}

An overview of the field of Analytical Cartography and conceptual topics in it can be found in a special issue of Cartography and Geographic Information Science (Moellering, ed. 2000a) on "The Nature of Analytical Cartography". A more specific article by Moellering (2000b) in that issue examines and discusses the scope and conceptual content of the field of Analytical Cartography in some detail. His article identifies more than 20 conceptual topics with 200 supporting scientific references to the literature in the field. A partial list of these topics can be seen as:

- Geographical Map Transformations

- Real/Virtual Map Transformations

- Deep \& Surface Structure

- Nyerges Data Levels

- Spatial Primitive Objects

- Topological Data Structures

- The Sampling Theorem

- Spatial Semantics \& Ontology

- Spatial Neighborhood Operators

- Spatial Frequencies

- Spatial Fourier Theory

- Spatial Information Theory

- Spatial Fractal Theory

- Critical Surface Features

- Warntz Networks

- Polygon Overlay Analysis

- Irregular Neighborhood Operators

- Resolution Elements - Resels

- Volumetric Pycnophlactic Transformations

- Map Generalization - 1,2,3-D

- Shape Analysis

- Spatial Data Models

- Analytical Visualization

- Metadata and Spatial Data Standards

- Spatial Semantics and Ontology

The above topics are a sample of the myriad of topics that have been developed over the last several decades in Analytical Cartography. A discussion of research needs in analytical cartography was discussed at the ICC 2001 Beijing meetings by Moellering (2001).

Interestingly, one of the most recent new topics, spatial semantics and ontology, has seen a tremendous development and use in the last decade. This is direct evidence of the continuing growth of the concepts and theory in analytical cartography.

\section{Analytical Cartography Material for the ICA Body of Knowledge}

The discussion above presents a very wide range of concepts and theory from analytical cartography that should be included in the proposed ICA Body of Knowledge for Cartography. All of them originate in the Deep Structure of the field, and many of them have Surface Structure cartographic realizations in the form of maps or visualizations.

This author has advocated that the ICA develop a Body of Knowledge document for Cartography since the Paris ICC 2011 Congress. Finally, it appears that the ICA has seen the light and is in the beginning stages of organizing to develop a BoK for Cartography (Usery, 2019) that will be discussed at ICC 2019 in Tokyo. This is welcome news for the entire field of cartography.

\section{Summary and Conclusions}

The need for a Body of Knowledge for Cartography was realized in the first decade of the 21 st century, and was proposed by Moellering at the ICC 2011 meetings in Paris. Since that time the idea has been debated and slowly developed to the point where the ICA is on the cusp of formally deciding to move forward with such a project. The discussion above leads to three major conclusions:

- There has been an Implicit BoK for cartography for many decades, which by its nature is disjoint and highly varied. Now is the time for the ICA to move forward to develop an Explicit Body of Knowledge for Cartography that includes all conceptual and theoretical aspects of the field.

- The concepts and theory included in the ICA BoK should come from all three operational paradigms in the field: Mapping \& Visualization, Communication, and Analytical. This should provide a balanced BoK for the field.

- Because of its relative newness in the field, additional attention should be paid to the analytical concepts and theory, some of which are not known so widely, but clearly are part of the conceptual analytical base of the field.

\subsection{Future Work}

Given the discussion above, it is clear that the ICA should move ahead with a working group to initiate and carry out the task to design and develop a Body of Knowledge for the field of Cartography. This work should include all conceptual aspects of the field including conceptual, conventional, perceptual, virtual, production, quantitative and analytical concepts. In order 
to develop a comprehensive BoK for Cartography, a wide range of researchers in the field should be involved in this work. Although that might take more time and effort than initially expected, it is a good strategy to ensure that the BoK for Cartography will be comprehensive and include all aspects of the field.

\section{References}

Buchroithner, M., and P. Fernández (2011) "Cartography in the Context of Sciences: Theoretical and Technological Considerations", Cartographic Journal, Vol 48, (1), pp. 4- 10.

Moellering, H., ed. (2000a) The Nature of Analytical Cartography", Cartography and Geographic Information Science, 27(3), pp. 187-188.

Moellering, H., (2000b), "The Scope and Conceptual Content of Analytical Cartography", Cartography and Geographic Information Science, Vol. 27(3), pp. 205 223.

Moellering, H., (2001) "Research Needs in Analytical Cartography", Proceedings of the 20th International Cartographic Conference, Beijing, China, Vol.5, pp. 3109-3118.

Moellering, H., (2011) "The ICA Research Agenda: Review and Comments", ", Proceedings of the 25th International Cartographic Conference, Paris, France.

Moellering, H., (2012) "The International Cartographic Association Research Agenda: Review, Perspectives, Comments and Recommendations", Cartography and Geographic Information Science, Vol. 39 (1), pp. 61-68.

Nyerges, T., (1991), "Analytical Map Use", Cartography and Geographic Information Systems, 18(1), pp. 11-22.

Tobler, W., (1961), Map Transformations of Geographic Space, Unpublished Ph. D. dissertation, University of Washington, $183 \mathrm{pp}$.

Tobler, Waldo, (1976), "Analytical Cartography", American Cartographer, 3(1), pp. 21-31.

Usery, Lynn, (2019) personal communication.

Virrantaus, K., and D. Fairbairn, M.-J. Kraak, (2009) "ICA Research Agenda on Cartography and GI Science", Cartographic Journal, Vol 46 (2), pp. 63-75.

Wikipedia (2018) Definition of Body of Knowledge, URL:

https://en.wikipedia.org/wiki/Body_of_knowledge 